\title{
ARTICLE \\ Purification of surfactin compounds produced by a Bacillus subtilis strain
}

\author{
Attila Bartal, Henriett Hunkár, Gábor Endre, Mónika Vörös, Csaba Vágvölgyi, András \\ Szekeres*
}

Department of Microbiology, Faculty of Science and Informatics, University of Szeged, Szeged, Hungary

\begin{abstract}
Surfactins are lipopeptide-type biosurfactants produced mainly by Bacillus species containing a peptide loop of seven amino acids and a hydrophobic fatty acid chain. These molecules exhibit various biological activities; therefore, their therapeutic and environmental applications are in the focus worldwide. In our work, a multi-step purification and separation process was developed to isolate surfactins from the ferment broth of $B$. subtilis SZMC 6179J strain. The process incorporates normal phase flash chromatography for pre-purifying the crude extract and two consecutive reverse phase HPLC separations for the isolation of the various surfactin molecules. The determination of the relative amounts of lipopeptides both in the crude extract and in each fraction of every separation step were carried out by HPLC-HESI-MS examinations. The ratio of surfactins in the crude extract was $21.35 \%$, but after the preparative flash chromatographic separation the relative amount of surfactins was observed to be $30.44 \%$. The preparative HPLC purification step resulted $85.39 \%$ purity of the surfactins. Nine different surfactin variants were isolated and identified from the fractions of this final semi-preparative HPLC purification, out of which three compounds were completely purified, and three others were detected in relative amounts of more than $95 \%$ in some fractions.

Acta Biol Szeged 64(2):121-128 (2020)
\end{abstract}

\author{
KEY WORDS \\ Bacillus subtilis \\ HPLC \\ preparative purification \\ surfactin
}

\author{
ARTICLE INFORMATION \\ Submitted \\ 20 October 2020 \\ Accepted \\ 14 November 2020 \\ *Corresponding author \\ E-mail: andras.j.szekeres@gmail.com
}

\section{Introduction}

Biosurfactants are natural products of various microorganisms such as bacteria, yeasts and fungi. They are constituted of peptides, saccharides or lipids or their combinations (Ward 2010). Biosurfactants have an amphiphilic nature due to possessing both hydrophilic and hydrophobic moieties, thus making these compounds able to lower the surface tensions of water/air, oil/air, or the oil/water interfaces (Santos et al. 2016). Most biosurfactants are either anionic or neutral, and few of them are cationic. The hydrophobic moieties of these molecules are made up of fatty acids, hydroxy fatty acids, or $\alpha$-alkyl$\beta$-hydroxy fatty acids possessing greater chain lengths. The hydrophilic parts can be carbohydrates, amino acids, cyclic peptides, phosphates, carboxylic acids, or different alcohols (Mulligan 2005). According to a recent study there are several causes for microorganisms to produce biosurfactants, mainly the necessity of changing the surface or interfacial properties of cells or the surrounding environment. These changes are mostly facilitating the formation and development of fruiting bodies and biofilms, the gliding movement or the swarming of cells. The amount of lipopeptides produced by the bacteria can be influenced by altering the culture conditions, for example with the application of different carbon sources and metal ions (Bartal et al. 2018) or the $\mathrm{pH}$ control of the ferment broth (Czinkóczky and Németh 2020). These compounds play an important role in controlling cell development, therefore they have various therapeutic activities including anti-microbial and anti-tumour effects (Mukherjee and Das 2010). Biosurfactants also possess important properties, such as low toxicity and specificity, moreover they are relatively easy to prepare. These bioactive compounds have attracted considerable interest during the past decade. Due to their characteristics, there were several attempts for their industrial applications, for example the bioremediation of organic chemicals, petroleum and petrochemicals, or their utilization as agrochemicals and fertilizers in agriculture. There are experiments for applications regarding food industry, cosmetics manufacturing, pharmaceuticals and detergents. Their attributes also propose the possibility for using biosurfactants as emulsifiers, demulsifiers, wetting agents, foaming agents and spreading agents (Volkering et al. 1997).

Lipopeptides are a wide group of microbial biosurfactants, consisting of a short linear or cyclic oligopeptide 
linked to a fatty acid chain. They are produced by a high variety of microorganisms such as fungi, including Aspergillus, and several different bacteria, for example Pseudomonas, Streptomyces, and Bacillus. These compounds claimed the most attention due to the discovery of their antimicrobial, immunosuppressive, antitumor, and high surface activities (Cameotra and Makkar 2004; Gross and Loper 2009; Pirri et al. 2009; Raaijmakers et al. 2010). The proposed antimicrobial mechanism of lipopeptides is forming pores in the cellular membrane, resulting in an imbalance in ion flux leading to eventual cell death (Bender et al. 1999; Baltz 2009; Raaijmakers et al. 2010).

In 1968, Arima et al. isolated a substance in the form of needle-shaped white crystals from Bacillus subtilis and they named it "surfactin" due of its strong surface activity (Arima 1968). In addition to this property, surfactin was also proved to inhibit fibrin clot formation. Arima characterized surfactin as lipopeptide by its nature and its amino acids composition was also determined (Arima 1968). During 1969 several studies have been made to elucidate the full structure of surfactin (Kakinuma et al. 1969a, 1969b). The firstly described surfactin compound consists of a hydrophobic fatty acid "tail part" and a hydrophilic cyclic heptapeptide "head part" linked together by a lactone bridge (Bonmatin et al. 2003; Kakinuma et al. 1969b). Subsequent studies in the next decades revealed that surfactins have more variants having different fatty acid chain lengths and various changes in their amino acid sequences (Jenny et al. 1991; Lin et al. 1994; Yakimov et al. 1995; Kecskeméti et al. 2018). Surfactins have an acidic nature and can be dissolved in alkaline water and in polar organic solvents like methanol, ethanol, acetone, ethyl acetate, chloroform, methylene chloride and acetic acid, although it is insoluble in water, petroleum ester, hexane and other non-polar organic solvents. In its solid state, surfactin forms white, needle-shaped crystals, and it shows a powerful surface activity, exceeding that of sodium lauryl sulphate. Surfactin can lower the water's surface tension from $72 \mathrm{mN} / \mathrm{m}$ to $27 \mathrm{mN} / \mathrm{m}$ at very low concentrations $(-0.005 \%)$. UV spectroscopic observations show that surfactin has no absorption in the range of $230-400$ nm (Arima et al. 1968).

Recently there is a growing interest in studying surfactins, triggered by the increasing evidence for their potential as medical therapeutic agents, especially as antiviral, antimicrobial, anticancer, hemolytic, blood anticoagulant, and fibrinolytic agents (Al-Ajlani et al. 2007). Surfactin is produced mainly by the Gram-positive, endosporeforming bacteria Bacillus subtilis (Peypoux et al. 1999), as well as B. pumilus, B. amyloliquefaciens, B. licheniformis, and B. mojavensis (Alvarez et al. 2011; Chen et al. 2015).

In the past decades there has been a growing scientific and industrial attention towards using naturally produced biosurfactants instead of their chemically synthesized counterparts. The main reason behind this is the advantages of these natural compounds such as their low toxicity, easier biodegradability and possible specific activity amongst extreme conditions. While increasing number of the different surfactin variants reported, there is a lack of information regarding their exact structure and the possible differences in their biological activities. Therefore, the aim of our work was the purification of the different surfactin variants by various separation techniques for their further structural elucidation and to serve possibilities for the detailed examinations of their biological activities.

\section{Materials and Methods}

\section{Culture conditions and sample preparation}

The examined Bacillus subtilis strain SZMC 6179J was previously isolated from tomato rhizosphere and its antagonistic properties were characterized and reported by Vágvölgyi et al. (2013).

For the surfactin production, a liquid ferment broth was applied according to the method described by Besson et al. (1987). It contained $10 \mathrm{~g} / \mathrm{L}$ glucose, $5 \mathrm{~g} / \mathrm{L}$ glutamic acid, $1 \mathrm{~g} / \mathrm{L} \mathrm{KH}_{2} \mathrm{PO}_{4}, 1 \mathrm{~g} / \mathrm{L} \mathrm{K}_{2} \mathrm{HPO}_{4}, 1 \mathrm{~g} / \mathrm{L} \mathrm{KCl}, 500 \mathrm{mg} / \mathrm{L}$ $\mathrm{MgSO}_{4} \times 7 \mathrm{H}_{2} \mathrm{O}, 5 \mathrm{mg} / \mathrm{L} \mathrm{FeSO}_{4} \times 7 \mathrm{H}_{2} \mathrm{O}$, and $160 \mu \mathrm{g} / \mathrm{L}$ $\mathrm{CuSO}_{4} \times 5 \mathrm{H}_{2} \mathrm{O}$. Bacteria were inoculated into $4 \times 1 \mathrm{~L}$ medium in four 2-L Erlenmeyer flasks, then incubated on a rotary shaker at $120 \mathrm{rpm}$ for 5 days at $25^{\circ} \mathrm{C}$. After this, the cells were removed from the ferment broth by centrifugation $\left(4{ }^{\circ} \mathrm{C}, 8000 \mathrm{rpm}, 15 \mathrm{~min}\right)$ using aliquots in $50 \mathrm{~mL}$ Falcon tubes. Peptides were precipitated from the supernatant acidified to $\mathrm{pH} 2$ by the addition of hydrochloric acid (VWR Chemicals, Hungary). After an overnight incubation at $4{ }^{\circ} \mathrm{C}$, the acidified ferment broth was centrifuged $\left(4^{\circ} \mathrm{C}, 8000 \mathrm{rpm}, 15 \mathrm{~min}\right)$, the supernatant was discarded, and the collected pellet was resolved in 50 $\mathrm{mL}$ methanol. The remaining particulates were removed by centrifugation, and after the addition of $1 \mathrm{~g}$ silica gel the solvent methanol was evaporated by a rotational vacuum evaporator.

\section{Separation methods}

\section{Flash chromatography}

The crude extract from the B. subtilis strain SZMC 6179J containing the produced surfactin compounds was pre-purified for separation by a CombiFlash EZ Prep Streamline Flash and Preparative HPLC Chromatograph (Teledyne ISCO, USA). The sample evaporated on silica gel (Molar Chemicals, Hungary) was loaded into a RediSep 25 g cartridge (Teledyne ISCO, USA), while the silica gel used for 
the purification was loaded into a Biotage $50 \mathrm{~g}$ cartridge (Teledyne ISCO, USA). The applied elution program was isocratic using two solvents: $70 \%$ toluene (eluent A, Molar Chemicals, Hungary) and 30\% methanol (eluent B, Molar Chemicals, Hungary) of five column volumes $(500 \mathrm{~mL})$. The flow rate was $40 \mathrm{~mL} / \mathrm{min}$ and $20 \mathrm{~mL}$ fractions were collected into $30 \mathrm{~mL}$ test tubes leading to 25 fractions altogether and the length of the run was $12.5 \mathrm{~min}$.

\section{Preparative and semi-preparative HPLC methods}

After the flash chromatographic run, a preparative HPLC purification was carried out to further cleanse the sample from contaminants for facilitating the actual isolation of the different surfactin variants by a semi-preparative HPLC separation. Both of them were performed by the aforementioned CombiFlash EZ Prep instrument (Teledyne ISCO, USA) in reverse phase and with the same solvents: eluent A was water and eluent $B$ was a mixture of acetonitrile/methanol (1:1, V/V \%, Molar Chemicals, Hungary), both solvents supplemented with $0.1 \%$ acetic acid (VWR International, Hungary).

The preparative step was achieved on a Phenomenex Gemini-NX (Phenomenex, USA) column $(5 \mu, \mathrm{C} 18,100$ $\times 21.20 \mathrm{~mm}$ ) using the following gradient elution time program: $30 \%$ of eluent B for $2 \mathrm{~min}$ followed by a sheer increase to $75 \%$ in $2 \mathrm{~min}$. Then a slower, linear increase to $95 \%$ follows ending at $30 \mathrm{~min}$, where this rate was held for $15 \mathrm{~min}$, then decreased to the initial $30 \%$ in $2 \mathrm{~min}$ and remained constant until the pressure stabilized ending the run of $60 \mathrm{~min}$ in total. The flow rate was $10 \mathrm{~mL} / \mathrm{min}$, the injection volume was $5 \mathrm{~mL}$ and $20 \mathrm{~mL}$ fractions were collected into $30 \mathrm{~mL}$ test tubes, having 30 fractions in total.

The semi-preparative isolation was executed on a BioBasic-18 (Thermo Scientific, USA) column ( $5 \mu$, C18, $250 \times 10 \mathrm{~mm}$ ) with an isocratic elution of $80 \%$ eluent B for $90 \mathrm{~min}$. The flow rate was $5 \mathrm{~mL} / \mathrm{min}$, the injection volume was $1 \mathrm{~mL}$ and fractions collected every $5 \mathrm{~mL}$ into $13 \mathrm{~mL}$ test tubes, resulting in 90 fractions.

\section{Analytical HPLC-MS methods}

The purity of the crude extracts and the fractions of the flash chromatographic and preparative HPLC separations, as well as the identification and relative quantitative examination of the semi-preparative HPLC fractions were carried out by HPLC-HESI-MS measurements. The applied instrument was a Nexera XR HPLC (Shimadzu Corporation, Japan) coupled with a TSQ Quantum Access triple quadrupole mass spectrometer (Thermo Scientific, USA). Both the quantitative and qualitative acquisitions implemented the eluents of the preparative and semipreparative HPLC isolations described above, and also the following reverse phase gradient elution time program was utilized in both cases: $5 \%$ eluent B for $2 \mathrm{~min}$, increased to
$80 \%$ in the following $2 \mathrm{~min}$, then gradually raised to $95 \%$ at $24 \mathrm{~min}$. This rate was held for $9 \mathrm{~min}$, then dropped to $5 \%$ in $2 \mathrm{~min}$, followed by a $5 \mathrm{~min}$ long equilibration stage, ending the run of $40 \mathrm{~min}$ in total. The flow rate was 0.2 $\mathrm{mL} / \mathrm{min}$ and the column heater temperature was $30^{\circ} \mathrm{C}$. The applied column was a Gemini-NX $(3 \mu, \mathrm{C} 18,150 \times 2$ $\mathrm{mm})$. The injection volume was $10 \mu \mathrm{L}$.

Both the purity checking, and the identifying measurements were carried out with heated electrospray ionization (HESI) ion source and in positive polarity. The spray voltage was $+4000 \mathrm{~V}$, the vaporizer temperature was 285 ${ }^{\circ} \mathrm{C}$, the capillary temperature was $350{ }^{\circ} \mathrm{C}$, the sheath gas pressure was 10 psi and the auxiliary gas pressure was 15 psi. The examinations of the purified fractions were achieved in full scan mode set between $900-1600 \mathrm{~m} / z$ values with the scan time of $1 \mathrm{sec} / \mathrm{scan}$. The identification of the different surfactin variants were performed in single reaction monitoring (SRM) mode with a collision energy of $60 \mathrm{~V}$. The $\mathrm{m} / z$ values of sodiated surfactin molecules were set as parent ion masses $(\mathrm{m} / z$ 1016.7, 1030.7, $1044.7,1058.7,1072.7,1086.7,1100.7,1114.7)$ and for every parent ion, the first two internal fragment ions of every natural surfactin variant were set as a daughter ion $(\mathrm{m} / \mathrm{z}$ 580.7, 594.7, 608.7, 622.7, 679.7, 693.7, 707.7, 721.7, 735.7) (Kecskeméti et al. 2018). Simultaneously with the SRM analyses, selected ion monitoring (SIM) mode was also used for the relative quantitative measurement of the detected isoforms and homologues, examining the parent ion $\mathrm{m} / z$ values listed above. With these two modes, 80 different scan events ran parallel, each for a scan time of $0.1 \mathrm{sec} / \mathrm{scan}$.

\section{Results}

\section{Analysis of crude surfactin extract}

After the extraction of the lipopeptides from the ferment broth, the initial purity of the crude extract was measured by HPLC-HESI-MS examination in full MS mode (Fig. 1A). By calculating the integrated area of all peaks of the total ion chromatogram (TIC) of the sample and the extracted ion chromatogram (EIC) of all $\mathrm{m} / z$ values belonging to surfactins, which eluted from $20 \mathrm{~min}$ to 28 min, their ratio was observed in the crude extract to be $21.35 \%$. As it can be seen, many contaminants appeared mostly at the lower retention time region, therefore the inclusion of a pre-purification step was recommended before loading our sample on a preparative HPLC column in large amounts. For that purpose, flash chromatographic separation was applied. 


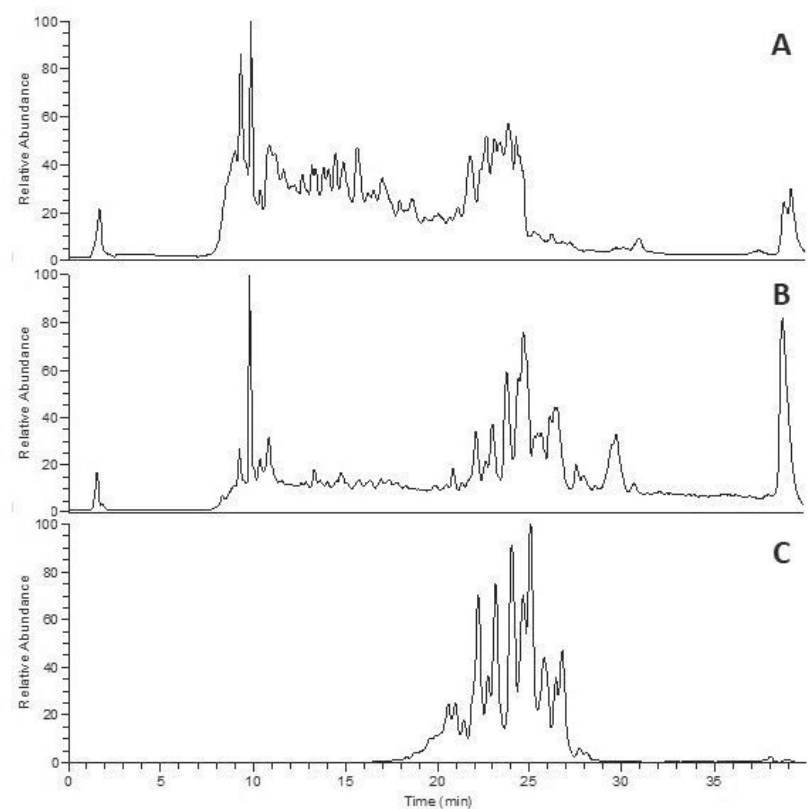

Figure 1. The total ion chromatogram of surfactins in the crude extract $(A)$, in the 6th fraction of the flash chromatographic separation (B) and the 10th fraction of the preparative HPLC separation (C).

\section{Flash chromatographic and preparative HPLC separa- tion}

Every fraction of the flash chromatographic separation was collected and their surfactin content and purity were measured by HPLC-HESI-MS technique in full MS mode. Although it could be observed that the relative amount of contaminants decreased considerably, the peak around the ten minute mark still exceeds the intensity of surfactins (Fig. 1B). Based on the calculation of the integrated peak areas of both the TICs and the EICs of all fractions the relative amount of surfactins and their purity were determined (Fig. 2). Based on these calculations, fractions $4-7$ were pooled, while the rest were discarded. The ratio of surfactins in this pooled sample was $30.44 \%$.

To further purify the sample from impurities, a preparative HPLC isolation was performed on the sample containing the combined fractions $4-7$. Following the preparative HPLC separation, the resulting 30 fractions were collected and their relative surfactin content and its purity were measured also by HPLC-HESI-MS examinations. Comparing the chromatograms (Fig. 1), it can be outright seen that most of the contaminants were separated from surfactins, all the peaks possessing lower retention times disappeared, only some minor impurities can be observed among the peaks of surfactins, suggesting that these compounds bear similar affinity to the stationary phase, therefore eluted along with the lipopeptides to be isolated (Fig. 1C). After the evaluation of all frac-

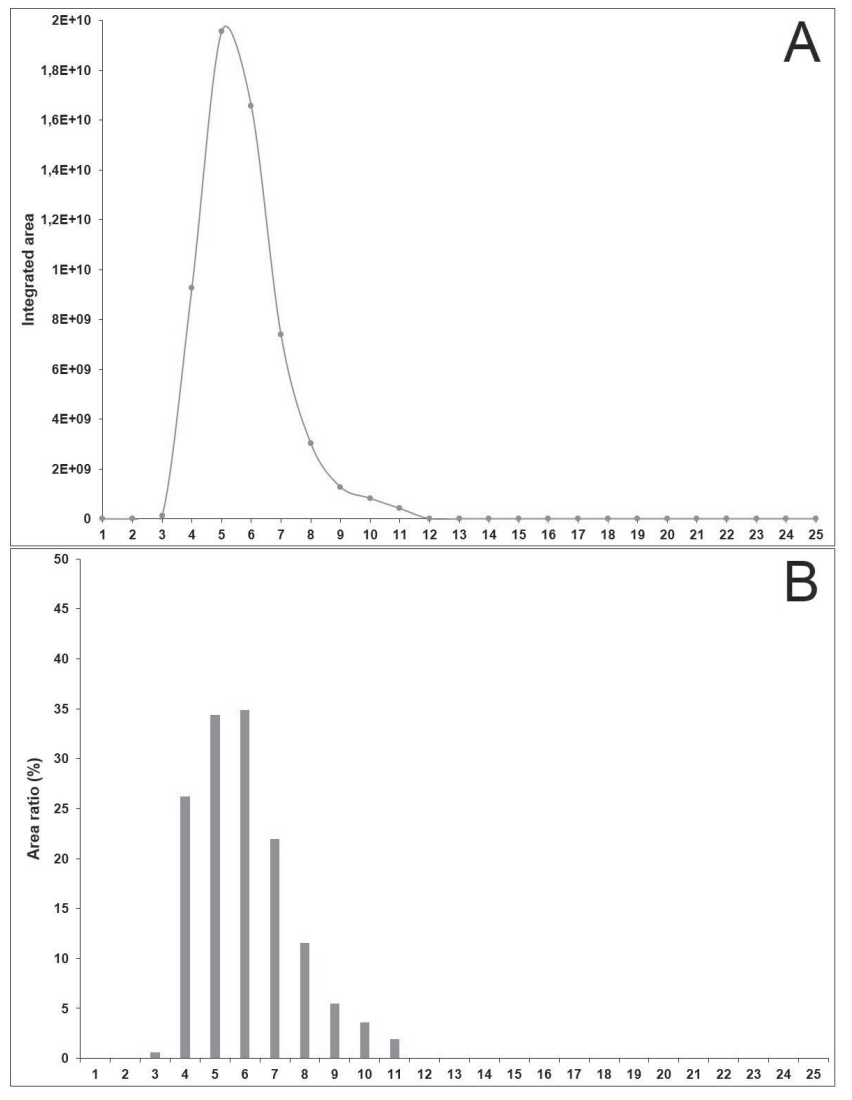

Figure 2. The intensities (A) and purities (B) of each fraction of the preparative flash chromatographic separation.

tions, the intensities and area ratios of surfactins were calculated (Fig. 3). For the most efficient isolation of the different surfactin variants, the combined fractions are recommended to be as pure as possible, while the sample should contain relatively high amount of surfactins. For these considerations, fractions 9 - 13 were merged and the rest of those that contain these lipopeptides were stored for subsequent purification. The final surfactin ratio of the pooled sample was $85.39 \%$.

\section{Semi-preparative HPLC separation}

As a final step, a semi-preparative HPLC separation was carried out for the isolation of the different surfactin homologues and isoforms. The identification of the detected surfactin variants and their relative amounts were measured in all the fractions by HPLC-HESI-MS technique, in SRM and full MS modes, respectively (Fig. 4).

By the examination of the $\mathrm{MS}^{2}$ spectra of all fractions of the semi-preparative HPLC separation, 9 molecules were identified altogether, with 4 different amino acid sequences ([Sur], [Val2], [Val7], [Val2,7]) and with 3 different fatty acid chain lengths (C13-C15). Out of the 9 variants, only C13-[Val2], C15-[Val7] and C15-[Sur] were 


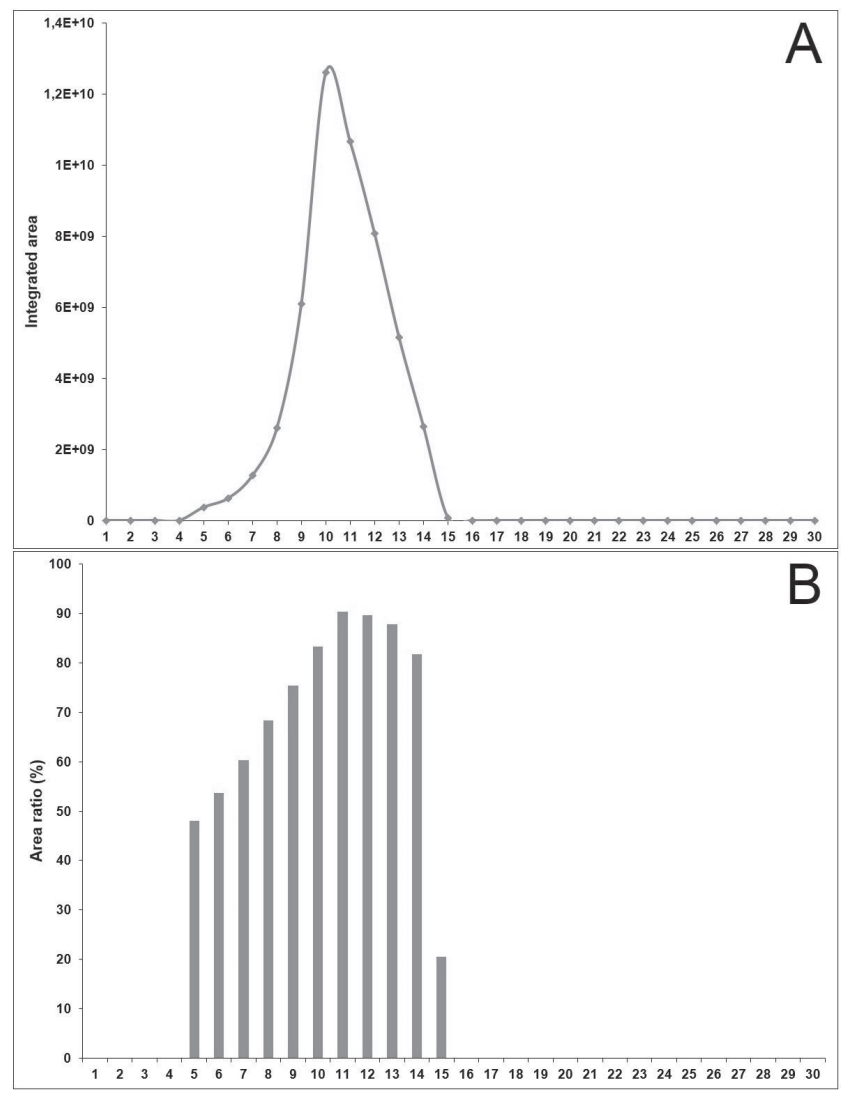

Figure 3. The intensities (A) and purities (B) of each fraction of the preparative HPLC separation.

detected with more than $99 \%$ ratio in a fraction. In the cases of C15-[Val7], C14-[Val2,7], C13-[Sur], C14-[Val2], C14-[Val7] and [C14-Sur], their most pure fractions had ratios of $98.55 \%, 43.66 \%, 95.34 \%, 53.81 \%, 98.69 \%$ and $77.54 \%$, respectively. The relative amounts of these molecules were also observed in comparison to the total integrated peak areas of every variant in every fraction (Fig. 5). Among the 9 molecules, C14-[Val7] and C14-[Sur] were found in the highest relative amounts, in $21.40 \%$ and 20.81\%. They were followed by C14-[Val2,7], C15-[Val7], C15-[Sur] and C13-[Val7], with the area ratios of 12.13\%, $11.10 \%, 11.05 \%$ and $10.93 \%$, respectively. C13-[Sur] was observed in $7.12 \%$ of the total peak area, while C14-[Val2] had a ratio of $4.18 \%$ and $\mathrm{C} 13$-[Val2] had been found in the lowest relative amount, it only possesses $1,29 \%$ of the total surfactin amount detected in the fractions of the semi-preparative HPLC separation.

\section{The efficiencies of the different separation steps}

After each purification step the collected and merged fractions were evaporated and the weights of the dry matters were measured. The weights of surfactins and other impurities were calculated based on their respective peak

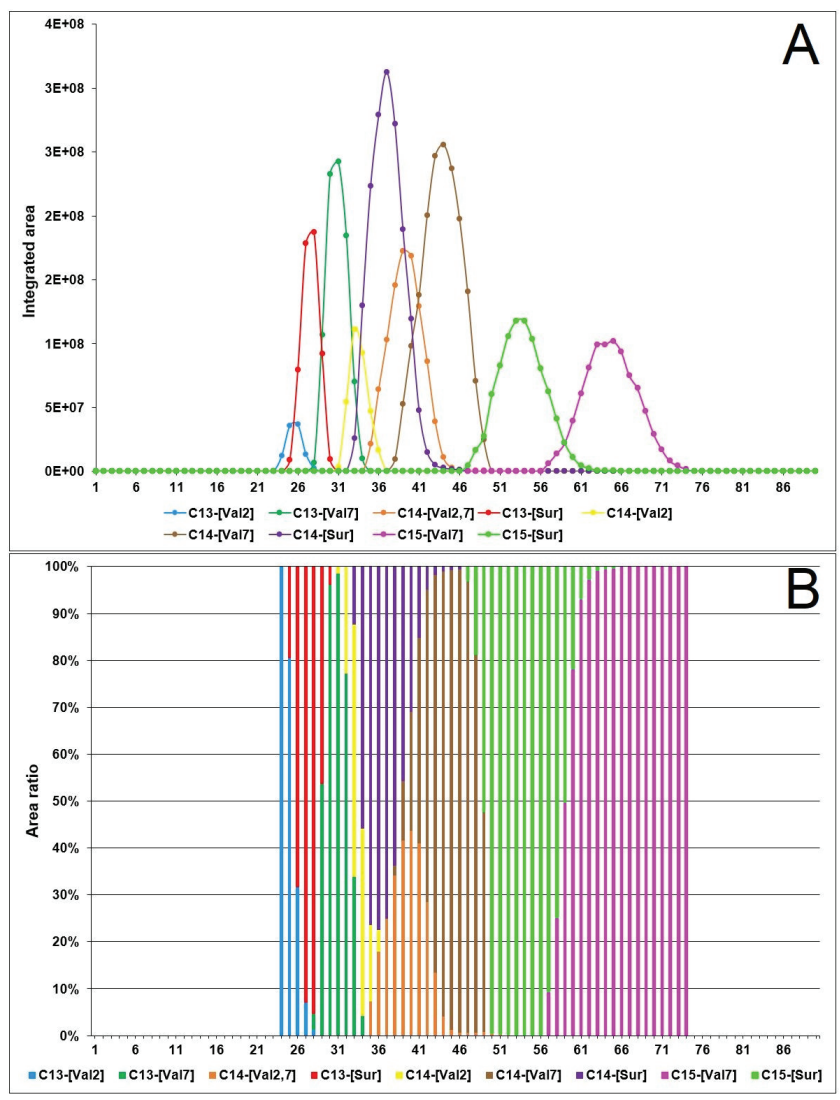

Figure 4. Fractogram of the identified surfactin variants (A) separated by semi-preparative HPLC technique and the ratios of surfactins in the fractions (B).

area ratios of the HPLC-HESI-MS analyses, giving the efficiencies of the different separation techniques (Fig. 6).

In terms of peak area ratios, $21.35 \%$ of the total area belonged to surfactins in the crude extract. It was only

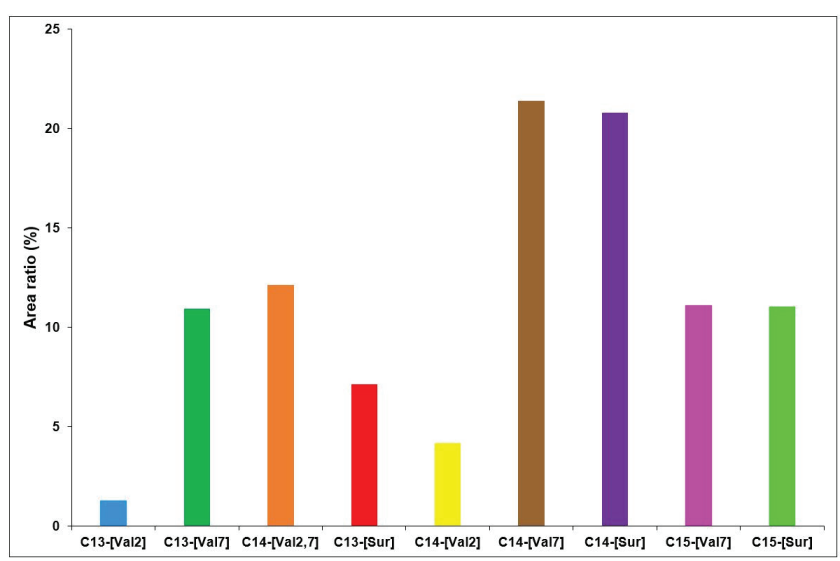

Figure 5. Ratios of the integrated peak areas of the different surfactin variants in fractions of the semi-preparative separation. 


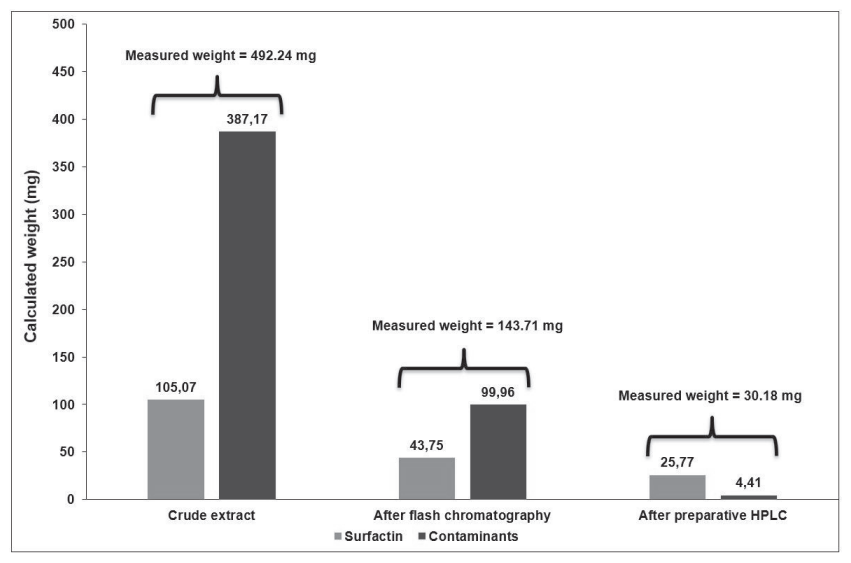

Figure 6. Measured weights of the dry matters after each separation step and the calculated weights of surfactins and the contaminants based on the peak area ratios of the HPLC-HESI-MS measurements.

increased to $30.44 \%$ after the flash chromatographic separation and the preparative HPLC purification technique raised the ratio of surfactins up to $85.39 \%$ altogether.

Based on these data and the measured weights of the dry matters, $74.18 \%$ of contaminants were removed by flash chromatography, and $95.59 \%$ of the remaining impurities were cleansed during preparative HPLC, leaving only $1.20 \%$ of the total amount of contaminants in the sample. On the other hand, the preparative flash chromatographic isolation removed $58.36 \%$ of surfactins as well, only to be further decreased by $41.10 \%$ by the preparative HPLC separation step. Thus, the calculated amount of surfactins remaining in the sample was only $24.53 \%$ that of the crude extract. This might be the explanation to the rather peculiar observation that only 9 different surfactin variants were detected in the fractions of the semi-preparative HPLC separation, although this strain is known to produce far more and in much higher variety of the different isoforms and homologues, which may have been lost during the purification process. Especially the loss of the molecules possessing longer fatty acid chains suggest that they were discarded among with the fractions collected later and having less area ratios than the ones being collected and merged.

In conclusion, the developed multi-step purification and separation of surfactins proved to be successful, more than $98 \%$ of the contaminants detected in the crude extract were removed and the C13-[Val2], C15-[Val7] and C15[Sur] variants were completely isolated from the rest of the molecules, while three others were detected in more than 95\% in some fractions. The efficiency of the separation however needs to be optimized, since around $75 \%$ of the calculated starting amount of surfactins were lost during the process, possibly including the homologues having more than 15 carbon atoms in their fatty acid chains. The flash chromatographic technique is especially in need of further development, subsequent repetitions of this step might be a suitable solution for decreasing the loss of surfactins and for increasing its purification potential.

\section{Discussion}

Surfactins have many potential applications, especially regarding therapeutic and environmental issues. The first example for the former was discovered by Arima et al. (1968); they observed that surfactin inhibits fibrin clot formation. Many other therapeutic activities were discovered later, e.g., antimycoplasmal, antibacterial and antiviral, anti-adhesive and anti-inflammatory effects (Vollenbroich et al. 1997; Kracht et al. 1999; Mulligan 2005; Heerklotz and Seelig 2006; Cho et al. 2006; Seydlová and Svobodová 2008). Furthermore, the use of surfactins as biosurfactans is an alternative to replace the chemically synthesized surfactant compounds. Biosurfactants are biodegradable and have lower toxicity (Kosaric 1992).

The length of the fatty acid chain and the amino acid sequence varies, resulting in numerous isoforms of the surfactins in previous works (Bonmatin et al. 2003). Although most of these variants were reported in the 1990s, our recent studies introduced novel surfactin molecules containing the following modifications: Val in the second amino acid position [Val2] (Bóka et al. 2016), aspartic acid 4-methyl ester at the fifth and Val in the seventh positions [AME5, Val7], leucine or isoleucine in the fourth and AME at the fifth positions [Lxx4, AME5] (Kecskeméti et al. 2018). Furthermore, it was also reported that the culture conditions of the producer strain could significantly influence the relative amounts of the individual members of surfactins produced allowing the selective production of certain groups of surfactins (Bartal et al. 2018).

However, the newly discovered surfactin variants were only characterized via spectrometric techniques and the confirmation of their exact structure will be important (for this the pure form of these compounds would be necessary in relatively large quantities). Furthermore, distinct surfactin variants could have different biological activities, as described by Aleti et al. (2016). They noted that two different surfactin variants with subtle structural differences had varying effects on bacterial biofilm formation on plant roots.

Therefore, in our work a multi-step purification and separation process was developed to isolate surfactins from the crude extract of the ferment broth of $B$. subtilis SZMC 6179J strain and to separate the different variants and homologues of this lipopeptide family. The method incorporates normal phase flash chromatography for 
pre-purifying the crude extract and two consecutive reverse phase high performance liquid chromatographic techniques; one with a preparative column to further cleanse the sample from contaminants, which was followed by a semi-preparative RP-HPLC for the isolation of the various surfactin molecules. The measurement of the relative amounts of lipopeptides in the crude extract and in each fraction of every step was carried out by HPLCHESI-MS examinations, as well as the identification of the different surfactin variants detected in the fractions of the semi-preparative HPLC separation.

The measured weight of the crude extract was 492.25 $\mathrm{mg}$ and the peak area ratio of surfactins in it was $21.35 \%$. After the preparative flash chromatographic separation, fractions $4-7$ were merged and kept for further purification. The weight of the dry matter found in these fractions was $143.71 \mathrm{mg}$ and the relative amount of surfactins was observed to be $30.44 \%$. Fractions 9 - 13 of the preparative HPLC purification step were collected together, their dry matter weighting $30.18 \mathrm{mg}$ altogether, while the integrated peak area of surfactins were $85.39 \%$ that of the total area of peaks detected in this sample, meaning that more than $98 \%$ of impurities detected in the crude extract were removed by these separation steps, however, about $75 \%$ of surfactins were also lost during the process.

Examination of the fractions of the semi-preparative HPLC isolation technique showed that altogether 9 different surfactin variants were isolated and identified, out of which 3 compounds were completely purified, and other 3 were detected in relative amounts of more than $95 \%$ in some fractions.

These results suggest that the developed multi-step method is applicable for the purification of surfactin isoforms and homologues for subsequent structural characterization by spectroscopic techniques, however, further optimization is necessary to increase the efficiency of the whole process.

\section{Acknowledgments}

This work was supported by the grant OTKA K-128659 from the Hungarian Scientific Research Fund providing infrastructure and research equipment.

\section{References}

Aleti G, Lehner S, Bacher M, Compant S, Nikolic B, Plesko M, Schuhmacher R, Sessitsch A, Brader G (2016) Surfactin variants mediate species-specific biofilm formation and root colonization in Bacillus. Environ Microbiol 18:2634-2645.
Al-Ajlani MM, Sheikh MA, Ahmad Z, Hasnain S (2007) Production of surfactin from Bacillus subtilis MZ-7 grown on pharmamedia commercial medium. Microb Cell Fact 6:17.

Alvarez F, Castro M, Príncipe A, Borioli G, Fischer S, Mori G, Jofré E (2012) The plant-associated Bacillus amyloliquefaciens strains MEP2 18 and ARP2 3 capable of producing the cyclic lipopeptides iturin or surfactin and fengycin are effective in biocontrol of sclerotinia stem rot disease. J Appl Microbiol 112:159-174.

Arima K, Kakinuma A, Tamura G (1968) Surfactin, a crystalline peptidelipid surfactant produced by Bacillus subtilis: isolation, characterization and its inhibition of fibrin clot formation. Biochem Biophys Res Commun 31:488-494.

Bartal A, Vigneshwari A, Bóka B, Vörös M, Takács I, Kredics L, Manczinger L, Varga M, Vágvölgyi C, Szekeres A (2018) Effects of different cultivation parameters on the production of surfactin variants by a Bacillus subtilis strain. Molecules 23:2675.

Bender CL, Alarcón-Chaidez F, Gross DC (1999) Pseudomonas syringae phytotoxins: mode of action, regulation, and biosynthesis by peptide and polyketide synthetases. Microbiol Mol Biol Rev 63:266-292.

Besson F, Chevanet C, Michel G (1987) Influence of the culture medium on the production of iturin $\mathrm{A}$ by Bacillus subtilis. J Gen Microbiol 133:767-772.

Bóka B, Manczinger L, Kecskeméti A, Chandrasekaran M, Kadaikunnan S, Alharbi NS, Vágvölgyi C, Szekeres, A (2016) Ion trap mass spectrometry of surfactins produced by Bacillus subtilis SZMC 6179J reveals novel fragmentation features of cyclic lipopeptides. Rapid Commun Mass Spectrom 30:1581-1590.

Bonmatin JM, Laprévote O, Peypoux F (2003) Diversity among microbial cyclic lipopeptides: Iturins and surfactins. Activity-structure relationships to design new bioactive agents. Comb Chem High Throughput Screen 6:541-556.

Cameotra SS, Makkar RS (2004) Recent applications of biosurfactants as biological and immunological molecules. Curr Opin Microbiol 7:262-266.

Chen WC, Juang RS, Wei YH (2015) Applications of a lipopeptide biosurfactant, surfactin, produced by microorganisms. Biochem Eng J 103:158-169.

Cho JY, Kim, Dae S, Park HJ, Lim JH, Yun HI, Park SC, Kim SK, Rhee MH (2006) A comparison of the antiinflammatory activity of surfactin A, B, C, and D from Bacillus subtilis. J Microbiol Biotechnol 16:1656-1659.

Czinkóczky R, Németh Á (2020) The effect of pH on biosurfactant production by Bacillus subtilis DSM10. Hung $\mathrm{J}$ Ind Chem 48:37-43.

Gross H, Loper JE (2009) Genomics of secondary metabolite production by Pseudomonas spp. Nat Prod Rep 26:1408-1446. 
Heerklotz H, Seelig J (2007) Leakage and lysis of lipid membranes induced by the lipopeptide surfactin. Eur Biophys J 36:305-314.

Jenny K, Käppeli O, Fiechter A (1991) Biosurfactants from Bacillus licheniformis: structural analysis and characterization. Appl Microbiol Biotechnol 36:5-13.

Kakinuma A, Hori M, Isono M, Tamura G, Arima K (1969a) Determination of amino acid sequence in surfactin, a crystalline peptidelipid surfactant produced by Bacillus subtilis. Agric Biol Chem 33:971-972.

Kakinuma A, Sugino H, Isono M, Tamura G, Arima K (1969b) Determination of fatty acid in surfactin and elucidation of the total structure of surfactin. Agric Biol Chem 33:973-976.

Kecskeméti A, Bartal A, Bóka B, Kredics L, Manczinger L, Shine K, Alharby NS, Khaled JM, Varga M, Vágvölgyi C, Szekeres A (2018) High-frequency occurrence of surfactin monomethyl isoforms in the ferment broth of a Bacillus subtilis strain revealed by ion trap mass spectrometry. Molecules 23:2224.

Kosaric N (1992) Biosurfactants in industry. Pure Appl Chem 64:1731-1737.

Kracht M, Rokos H, Ozel M, Kowall M, Pauli G, Vater J (1999) Antiviral and hemolytic activities of surfactin isoforms and their methyl ester derivatives. J Antibiot (Tokyo) 52:613-619.

Lin SC, Carswell KS, Sharma MM, Georgiou G (1994) Continuous production of the lipopeptide biosurfactant of Bacillus licheniformis JF-2. Appl Microbiol Biotechnol 41:281-285.

Mukherjee AK, Das K (2010) Microbial surfactants and their potential applications: An overview. In Sen R, Ed., Biosurfactants. Advances in Experimental Medicine and Biology. Vol 672. Springer, New York, 54-64.

Mulligan CN (2005) Environmental applications for biosurfactants. Environ Poll 133:183-198.
Peypoux F, Bonmatin JM, Wallach J (1991) Recent trends in the biochemistry of surfactin. Appl Microbiol Biotechnol 51:553-563.

Pirri G, Giuliani A, Nicoletto SF, Pizzuto L, Rinaldi AC (2009) Lipopeptides as anti-infectives: a practical perspective. Cent Eur J Biol 4:258-273.

Raaijmakers JM, De Bruijn I, Nybroe O, Ongena M (2010) Natural functions of lipopeptides from Bacillus and Pseudomonas: more than surfactants and antibiotics. FEMS Microbiol Rev 34:1037-1062.

Santos DK, Rufino RD, Luna JM, Santos VA, Sarubbo LA (2016) Biosurfactants: Multifunctional biomolecules of the 21st century. Int J Mol Sci 17:401.

Seydlová G, Svobodová J (2008) Review of surfactin chemical properties and the potential biomedical applications. Cent Eur J Med 3:123-133.

Vágvölgyi C, Sajben-Nagy E, Bóka B, Vörös M, Berki A, Palágyi A, Krisch J, Skrbić B, Durišić-Mladenović N, Manczinger L (2012) Isolation and characterization of antagonistic Bacillus strains capable to degrade ethylenethiourea. Curr Microbiol 66:243-250.

Volkering F, Breure AM, Rulkens WH (1997) Microbiological aspects of surfactant use for biological soil remediation. Biodegradation 8:401-417.

Vollenbroich D, Pauli G, Ozel M, Vater J (1997) Antimycoplasma properties and application in cell culture of surfactin, a lipopeptide antibiotic from Bacillus subtilis. Appl Environ Microbiol 63:44-49.

Ward OP (2010) Microbial biosurfactants and biodegradation. Adv Exp Med Biol 672:65-74.

Yakimov MM, Timmis KN, Wray V, Fredrickson HL (1995) Characterization of a new lipopeptide surfactant produced by thermotolerant and halotolerant subsurface Bacillus licheniformis BAS50. Appl Environ Microbiol 61:1706-1713. 